\title{
C-H bond dissociation enthalpies in norbornane. An experimental and computational study
}

\author{
Paulo M. Nunes`, Sílvia G. Estácio, Gustavo T. Lopes, Benedito J. \\ Costa Cabral, Rui M. Borges dos Santos, and José A. Martinho \\ Simões
}

Experimental Section

Materials: Benzene (Aldrich, HPLC grade, 99.9+ \%), was used as received. Norbornane (Aldrich, $98 \%$ ) was purified by triple sublimation in vacuum. Di-tertbutylperoxide (Aldrich) was purified according to a literature procedure. ${ }^{1} \quad$ Ortho-hydroxybenzophenone (Aldrich) was recrystallized twice from an ethanol-water mixture.

Photoacoustic calorimetry: The time-resolved photoacoustic calorimetry (TR-PAC) technique ${ }^{2}$ captures an acoustic wave generated by the sudden volume change that occurs when a solution is irradiated with a laser pulse, initiating a series of physico-chemical processes. The photoacoustic signal provides information on the intensity and the time profile of non-radiative energy released during these processes. Using a deconvolution analysis for the time dependence of the signal, both the magnitudes of each of the signal-inducing events as well as their lifetimes can be determined. ${ }^{3}$ The analysis involves first the normalization of the waveform for its respective absorbance and incident laser energy. Calculation of the observed heat fraction, $\phi_{\mathrm{obs}, \mathrm{i}}$, and the lifetime, $\tau_{\mathrm{i}}$, for each process is achieved by the deconvolution of the normalized waveform with a commercially available software. ${ }^{4}$ The parameter $\phi_{\mathrm{obs}, \mathrm{i}}$ is the observed fraction of photon energy released as heat which, when multiplied by the molar energy of the laser photons $\left(E_{\mathrm{m}}=N_{\mathrm{A}} h v\right)$, corresponds to the observed enthalpic change, $\Delta_{\mathrm{obs}} H_{\mathrm{i}}$.

In our scheme (see Scheme 1, in text) a two step sequential reaction occurs. The enthalpy of the first step (photochemical) and of the second (thermal) are given by eqs 1 and 2, respectively.

$$
\Delta_{\mathrm{r}} H_{1}=\frac{E_{\mathrm{m}}-\Delta_{\mathrm{obs}} H_{1}}{\Phi_{\mathrm{r}}}+\frac{\Delta_{\mathrm{r}} V_{1}}{\chi}
$$

$\Delta_{\mathrm{r}} H_{2}=\frac{-\Delta_{\mathrm{obs}} H_{2}}{\Phi_{\mathrm{r}}}+\frac{\Delta_{\mathrm{r}} V_{2}}{\chi}$

In these equations, $\Phi_{\mathrm{r}}$ represents the quantum yield of the first step. $\Delta_{\text {obs }} H_{\mathrm{i}}$ are calculated from the respective $\phi_{\mathrm{obs}, \mathrm{i}}$, obtained from the deconvolution analysis. $\phi_{\mathrm{obs}, \mathrm{i}}$ consists not only of a thermal contribution, due to the enthalpy of the reaction, but also of a reaction volume contribution, due to the differences between the partial molar volumes of the reactants and products. ${ }^{5}$ The latter leads to the introduction of a correction factor when calculating the reaction enthalpies. The correction term includes the reaction volume change, $\Delta_{\mathrm{r}} V_{\mathrm{i}}$, and the adiabatic expansion coefficient of the solution, $\chi$.

In this work, solutions of ca. $0.33 \mathrm{M}$ of di-tertbutylperoxide and ca. 3-4 $\mathrm{M}$ of norbornane in benzene were flowed through a quartz flow cell (Hellma 174-QS) and photolysed with pulses from a nitrogen laser (PTI PL 2300, $337.1 \mathrm{~nm}$, pulse width $800 \mathrm{ps}$ ). The incident laser energy was varied by using neutral density filters and the induced acoustic wave was detected by a piezoelectric transducer (Panametrics V101, 0.5 MHz) in contact with the bottom of the cell. The photoacoustic signals were measured by a digital oscilloscope (Tektronix 2430A), where the signal-to-noise ratio was improved by averaging 32 acquisitions. Waveforms were collected at various laser intensities to check for multiphoton effects. The apparatus was calibrated by carrying out a photoacoustic run using an optically matched (within typically $6 \%$ absorbance units at $337.1 \mathrm{~nm}$ ) solution of the photoacoustic calibrant ortho-hydroxybenzophenone in benzene. ${ }^{2}$ This solution does not include the peroxide but contains norbornane, in the same concentration as in the experiment. The sample waveform was deconvoluted with the calibration waveform using the software Sound Analysis by Quantum Northwest. ${ }^{4}$

From our TR-PAC experiments we obtained $\Delta_{\text {obs }} H_{2}=$ $59.2 \pm 2.5 \mathrm{kJmol}^{-1}$. The quantum yield for the photolysis of di-tert-butyl peroxide in benzene is $\Phi_{\mathrm{r}}=0.83$. Considering 
that this step is a metathesis reaction we may assume $\Delta_{\mathrm{r}} V_{2}=0 .{ }^{6}$ Hence, we derive $\Delta_{\mathrm{r}} H_{2}=-71.3 \pm 3.0 \mathrm{kJmol}^{-1}$.

Theoretical calculations

The complete basis-set composite schemes CBS-Q and CBS-QB33 ${ }^{7-9}$ were applied to determine the gas-phase $\mathrm{C}-\mathrm{H}$ BDEs of norbornane. Initially, optimized geometries and frequencies were determined at the B3LYP/cc$\mathrm{pVTZ}^{10,11}$ level. The choice of this approach was oriented by previous investigations, indicating that it is adequate for a reliable prediction of both closed-shell and openshell structures. Thermal corrections to $298.15 \mathrm{~K}$ were based on B3LYP/cc-pVTZ unscaled frequencies. All the calculations were carried out with the Gaussian-03 program and thermal corrections to $298.15 \mathrm{~K}$ were applied. $^{12}$

Cartesian coordinates (in ångströms) of B3LYP/ccpVTZ optimized geometries:

\begin{tabular}{|c|c|c|c|}
\hline $\mathrm{C}$ & -1.102201 & 0.772244 & 0.780759 \\
\hline $\mathrm{C}$ & 0.282000 & 0.186927 & 1.130320 \\
\hline $\mathrm{C}$ & 0.282000 & 0.186927 & -1.130320 \\
\hline $\mathrm{C}$ & -1.102201 & 0.772244 & -0.780759 \\
\hline $\mathrm{H}$ & -1.914161 & 0.178295 & 1.201632 \\
\hline $\mathrm{H}$ & -1.914161 & 0.178295 & -1.20163 \\
\hline $\mathrm{C}$ & 1.154979 & 0.765607 & 0.000000 \\
\hline $\mathrm{H}$ & 1.193605 & 1.856193 & 0.000000 \\
\hline $\mathrm{C}$ & 0.282000 & -1.315934 & 0.780760 \\
\hline $\mathrm{H}$ & -0.581284 & -1.832455 & 1.2016 \\
\hline $\mathrm{C}$ & 0.282000 & -1.315934 & -0.78076 \\
\hline $\mathrm{H}$ & -0.581284 & -1.832455 & -1.2016 \\
\hline $\mathrm{H}$ & 0.606706 & 0.402169 & 2.14714 \\
\hline $\mathrm{H}$ & 0.606706 & 0.402169 & -2.147 \\
\hline $\mathrm{H}$ & 2.174505 & 0.376461 & 0.00000 \\
\hline $\mathrm{H}$ & -1.205495 & 1.784882 & -1.1728 \\
\hline $\mathrm{H}$ & -1.205495 & 1.784882 & 1.1728 \\
\hline $\mathrm{H}$ & 1.174450 & -1.805462 & -1.17281 \\
\hline $\mathrm{H}$ & 1.174450 & -1.805462 & 1.17281 \\
\hline \multicolumn{4}{|c|}{ 7-norbornyl } \\
\hline $\mathrm{C}$ & -1.084360 & 0.792477 & 0.781560 \\
\hline $\mathrm{C}$ & 0.313727 & 0.227001 & 1.134239 \\
\hline $\mathrm{C}$ & 0.313727 & 0.227001 & -1.134239 \\
\hline $\mathrm{C}$ & -1.084360 & 0.792477 & -0.781560 \\
\hline $\mathrm{H}$ & -1.885789 & 0.184011 & 1.202403 \\
\hline $\mathrm{H}$ & -1.885789 & 0.184011 & -1.202403 \\
\hline $\mathrm{C}$ & 1.139723 & 0.771178 & 0.000000 \\
\hline $\mathrm{C}$ & 0.313727 & -1.289705 & 0.782171 \\
\hline $\mathrm{H}$ & -0.550976 & -1.803535 & 1.205324 \\
\hline $\mathrm{C}$ & 0.313727 & -1.289705 & -0.782171 \\
\hline $\mathrm{H}$ & -0.550976 & -1.803535 & -1.205324 \\
\hline $\mathrm{H}$ & 0.645173 & 0.445891 & 2.146486 \\
\hline
\end{tabular}

$\begin{array}{lrrr}\mathrm{H} & 0.645173 & 0.445891 & -2.146486 \\ \mathrm{H} & 2.212477 & 0.907934 & 0.000000 \\ \mathrm{H} & -1.199697 & 1.803572 & -1.171371 \\ \mathrm{H} & -1.199697 & 1.803572 & 1.171371 \\ \mathrm{H} & 1.207313 & -1.776080 & -1.173557 \\ \mathrm{H} & 1.207313 & -1.776080 & 1.173557\end{array}$

$\begin{array}{lrrr}\text { 1-norbornyl } & & & \\ \mathrm{C} & 1.260872 & 0.633237 & -0.610582 \\ \mathrm{C} & 0.000000 & 1.139760 & 0.132645 \\ \mathrm{C} & 0.000000 & -1.027216 & 0.529350 \\ \mathrm{C} & 1.258448 & -0.914896 & -0.301730 \\ \mathrm{H} & 1.218795 & 0.841711 & -1.679877 \\ \mathrm{H} & 1.224563 & -1.522827 & -1.204915 \\ \mathrm{C} & 0.000000 & 0.225054 & 1.388667 \\ \mathrm{H} & 0.890482 & 0.343017 & 2.005971 \\ \mathrm{C} & -1.260872 & 0.633237 & -0.610583 \\ \mathrm{H} & -1.218795 & 0.841711 & -1.679877 \\ \mathrm{C} & -1.258448 & -0.914896 & -0.301730 \\ \mathrm{H} & -1.224564 & -1.522827 & -1.204914 \\ \mathrm{H} & 0.000000 & 2.213916 & 0.314754 \\ \mathrm{H} & -0.890482 & 0.343017 & 2.005971 \\ \mathrm{H} & 2.148369 & -1.198827 & 0.260925 \\ \mathrm{H} & 2.160983 & 1.107130 & -0.217595 \\ \mathrm{H} & -2.148370 & -1.198826 & 0.260925 \\ \mathrm{H} & -2.160982 & 1.107131 & -0.217596\end{array}$

$\begin{array}{lrrr}\text { 2-norbornyl } & & & \\ \text { C } & -1.029275 & -1.000913 & -0.483824 \\ \text { C } & 0.263634 & -1.067497 & 0.362417 \\ \text { C } & -0.189726 & 1.150121 & 0.294826 \\ \text { C } & -1.370258 & 0.521156 & -0.509864 \\ \text { H } & -0.882136 & -1.413502 & -1.482330 \\ \text { H } & -1.435821 & 0.923811 & -1.519701 \\ \text { C } & 0.020300 & 0.065877 & 1.378503 \\ \text { C } & -0.862177 & -0.095983 & 1.999540 \\ \text { C } & 1.425870 & -0.482122 & -0.475414 \\ \text { H } & 1.509997 & -0.945971 & -1.463888 \\ \text { C } & 1.051455 & 0.973344 & -0.523285 \\ \text { H } & 1.433329 & 1.690337 & -1.235381 \\ \text { H } & 0.469909 & -2.051491 & 0.779307 \\ \text { H } & -0.386707 & 2.162977 & 0.640698 \\ \text { H } & 0.879406 & 0.263875 & 2.019809 \\ \text { H } & -2.319766 & 0.725886 & -0.010572 \\ \text { H } & -1.828302 & -1.573456 & -0.011836 \\ \text { H } & 2.390272 & -0.646281 & 0.024201\end{array}$

Table S1. Sum of electronic and thermal enthalpies (in hartrees) at $T=298.15 \mathrm{~K}$.

\begin{tabular}{llll}
\hline & CBS-Q & B3LYP/cc-pVTZ & CBS-QB3 \\
\hline norbornane & -273.376305 & -273.880756 & -273.375714 \\
7-norbornyl & -272.710955 & -273.218772 & -272.710444 \\
1-norbornyl & -272.706083 & -273.214408 & -272.706558 \\
2-norbornyl & -272.720834 & $-273,228799$ & $-272,720802$ \\
\hline
\end{tabular}


(1) Diogo, H. P.; Minas da Piedade, M. E.; Martinho Simões, J. A.; Nagano, Y. J. Chem. Thermodyn. 1995, 27, 597-604.

(2) Braslavsky, S. E.; Heibel, G. E. Chem. Rev. 1992, 92, 1381-1410.

(3) Small, J. R.; Libertini, L. J.; Small, E. W. Biophys. Chem. 1992, 42, 29-48.

(4) Sound Analysis, version 1.50D; Quantum Northwest: Spokane, WA, 1999.

(5) Hung, R. R.; Grabowski, J. J. J. Am. Chem. Soc. 1992, 114, 351353.

(6) Wayner, D. D. M.; Lusztyk, E.; Pagé, D.; Ingold, K. U.; Mulder, P.; Laarhoven, L. J. J.; Aldrich, H. S. J. Am. Chem. Soc. 1995, 117, 8737 8744.

(7) Ochterski, J. W.; Petersson, G. A.; Montgomery, J. A. J Chem Phys 1996, 104, 2598-2619.

(8) Montgomery, J. A.; Frisch, M. J.; Ochterski, J. W.; Petersson, G. A. J Chem Phys 1999, 110, 2822-2827.

(9) Montgomery, J. A.; Frisch, M. J.; Ochterski, J. W.; Petersson, G. A. J Chem Phys 2000, 112, 6532-6542.

(10) Becke, A. D. J. Phys. Chem. 1993, 98, 5648.

(11) Lee, C. T.; Yang, W. T.; Parr, R. G. Phys. Rev. B: Condens. Matter 1988, 37, 785 .

(12) Frisch, M. J.; Trucks, G. W.; Schlegel, H. B.; Scuseria, G. E.; Robb, M. A.; Cheeseman, J. R.; Montgomery, J., J. A.; Vreven, T.; Kudin, K. N.; Burant, J. C.; Millam, J. M.; Iyengar, S. S.; Tomasi, J.; Barone, V.; Mennucci, B.; Cossi, M.; Scalmani, G.; Rega, N.; Petersson, G. A.; Nakatsuji, H.; Hada, M.; Ehara, M.; Toyota, K.; Fukuda, R.; Hasegawa, J.; Ishida, M.; Nakajima, T.; Honda, Y.; Kitao, O.; Nakai, H.; Klene, M.; Li, X.; Knox, J. E.; Hratchian, H. P.; Cross, J. B.; Bakken, V.; Adamo, C.; Jaramillo, J.; Gomperts, R.; Stratmann, R. E.; Yazyev, O.; Austin, A. J.; Cammi, R.; Pomelli, C.; Ochterski, J. W.; Ayala, P. Y.; Morokuma, K.; Voth, G. A.; Salvador, P.; Dannenberg, J. J.; Zakrzewski, V. G.; Dapprich, S.; Daniels, A. D.; Strain, M. C.; Farkas, O.; Malick, D. K.; Rabuck, A. D.; Raghavachari, K.; Foresman, J. B.; Ortiz, J. V.; Cui, Q.; Baboul, A. G.; Clifford, S.; Cioslowski, J.; Stefanov, B. B.; Liu, G.; Liashenko, A.; Piskorz, P.; Komaromi, I.; Martin, R. L.; Fox, D. J.; Keith, T.; Al-Laham, M. A.; Peng, C. Y.; Nanayakkara, A.; Challacombe, M.; Gill, P. M. W.; Johnson, B.; Chen, W.; Wong, M. W.; Gonzalez, C.; Pople, J. A.; Gaussian, Inc., Wallingford CT: 2004. 